\title{
Entrelacs du savoir : la France et l'Histoire naturelle de la Norvège de Pontoppidan
}

\section{Simone Ochsner}

Traducteur : Pierre-Brice Stahl et Julien Collonges

\section{OpenEdition Journals}

Édition électronique

URL : https://journals.openedition.org/rbnu/1996

DOl : $10.4000 /$ rbnu. 1996

ISSN : 2679-6104

\section{Éditeur}

Bibliothèque nationale et universitaire de Strasbourg

\section{Édition imprimée}

Date de publication : 1 novembre 2013

Pagination : 52-61

ISBN : 9782859230517

ISSN : 2109-2761

\section{Référence électronique}

Simone Ochsner, «Entrelacs du savoir : la France et l'Histoire naturelle de la Norvège de

Pontoppidan », La Revue de la BNU [En ligne], 8| 2013, mis en ligne le 01 novembre 2013, consulté le 17 mai 2021. URL : http://journals.openedition.org/rbnu/1996 ; DOI : https://doi.org/10.4000/rbnu.1996

\section{c) (†) (2)}

La Revue de la BNU est mise à disposition selon les termes de la Licence Creative Commons Attribution - Pas d'Utilisation Commerciale - Partage dans les Mêmes Conditions 4.0 International. 


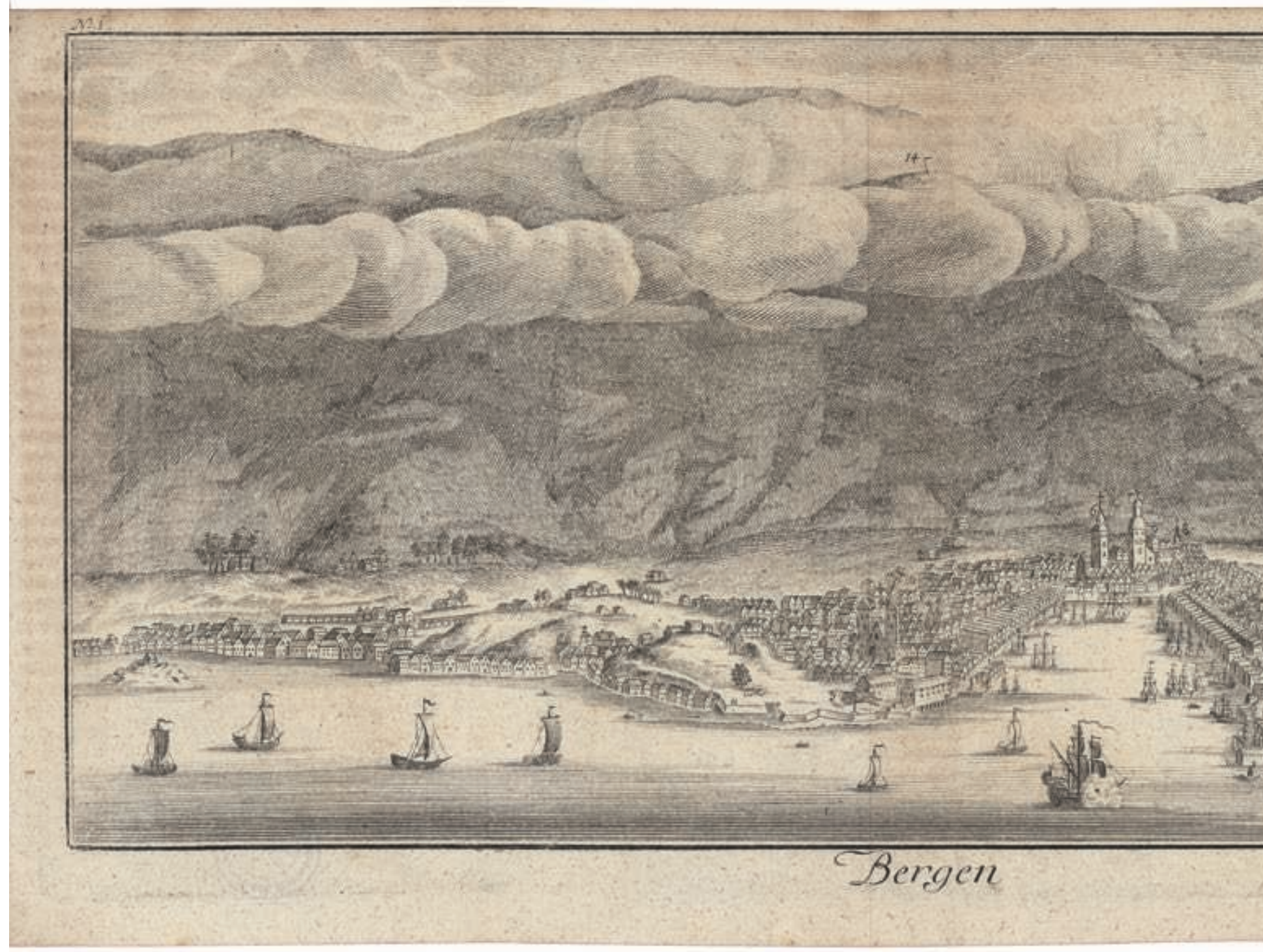

Gravure extraite du premier volume de l'Histoire naturelle de la Norvège (édition de Copenhague, 1753), représentant une vue de la ville de Bergen (coll. BNU) 
En examinant plus précisément le catalogue, l'on s'aperçoit qu'à l'exception de deux textes, dont la date d'entrée dans la collection n'est pas claire, tous ont intégré les fonds de la BNU entre 1872 et 1874 . Cette concentration sur une courte période est liée aux événements du 24 août 1870 , lorsque les deux bibliothèques principales de Strasbourg, " la bibliothèque du Séminaire protestant [...] et la bibliothèque municipale ${ }^{2}$, furent victimes d'un bombardement allemand qui entraîna en outre la capitulation de la ville. Devant l'émoi provoqué par la perte de ce patrimoine culturel, différentes mesures furent prises afin d'aider Strasbourg à se munir d'une nouvelle bibliothèque d'excellence. Parmi celles-ci, il faut citer les appels aux dons initiés par Karl-August Barack, un célèbre bibliothécaire allemand, qui rencontrèrent un très grand succès. Grâce à ces dons et à une dotation budgétaire importante et continue pendant toute la période allemande de l'histoire de l'établissement, il fut possible de célébrer dès le 9 août 1871 , soit moins d'un an après le grand incendie dévastateur, l'ouverture d'une bibliothèque de 200 ooo volumes ${ }^{3}$ qui prit alors le nom de "Kaiserliche Universitäts- und Landesbibliothek zu Strassburg "4. L'afflux d'argent pour les nouvelles acquisitions, ainsi que les dons et les dépôts, se poursuivirent les années suivantes; parmi l'énorme quantité de livres ainsi acquis figurent aussi les ouvrages de Pontoppidan. La manière dont ils trouvèrent le chemin de la BNU est variable : certains furent acquis grâce aux importants moyens financiers disponibles, pour d'autres il s'agit de dons d'institutions ou de particuliers, comme par exemple l'ouvrage Augustissima domus Oldenburgica in nuce augusta, offert par la Bibliothèque universitaire de Göttingen, ou la Kurz gefaste Reformations-Historie der dänischen Kirche, provenant de la bibliothèque privée du professeur de théologie strasbourgeois Edouard Cunitz. Les textes, publiés en allemand ou en latin, ou encore traduits du danois en allemand, furent imprimés à Copenhague, Brême, Hambourg, Lubeck et Leipzig, ce qui correspond bien à l'orientation linguistique d'une bibliothèque venant tout juste d'intégrer l'Empire allemand. Et si aujourd'hui la BNU est considérée comme la deuxième plus grande bibliothèque de France, cela est principalement dû à l'histoire singulière de sa création et à sa vaste collection d'ouvrages en langue allemande ${ }^{5}$.
Mais qui est l'auteur de ces huit textes qui, parmi tant d'autres, firent leur entrée à la BNU entre 1872 et $1874^{6}$ ? Erik Pontoppidan est né en 1698 à Aarhus. Il est considéré comme le représentant le plus éminent du piétisme d'Etat dano-norvégien au $18^{\mathrm{e}}$ siècle et comme l'un des premiers représentants des Lumières au royaume de Danemark et de Norvège. Après quelques années passées d'abord comme précepteur aux Pays-Bas, en Norvège et en Angleterre puis comme pasteur d'une paroisse au Danemark, il devint pasteur à la cour de cet Etat à la fin de l'année $1735^{7}$. Il fut un proche confident du roi piétiste Christian VI qui régna sur le Danemark et la Norvège de 1730 à 1746 . Pontoppidan doit principalement sa réputation à son ouvrage Sandhed til Gudfryktighed (1737), un commentaire du Petit catéchisme de Luther, qui est à l'origine de l'introduction dans la double monarchie, à la fin des années 1730, de l'enseignement obligatoire en vue de la confirmation. En 1738, il fut nommé professeur extraordinaire de théologie ${ }^{8}$, et devint membre par la suite de divers organismes. En 1742, en compagnie d'autres membres des premiers cercles gouvernementaux, il fonda la première société scientifique du royaume de Danemark et de Norvège, la « Videnskabernes Selskab ». Après la mort du roi Christian VI en 1746, il fut envoyé à Bergen, sur la côte ouest de la Norvège où, pendant la période même où son Histoire naturelle était publiée, il exerça les fonctions d'évêque. En 1755, Johan Ludvig Holstein, son mécène et protecteur de l'université de Copenhague, le fit revenir dans la capitale du royaume, où il devint le vicechancelier de cette même institution. Il occupa ce poste pendant près de dix ans, jusqu'à sa mort en 1764 .

Outre les textes historiques, politiques et d'histoire naturelle présents à la BNU de Strasbourg, et des traités religieux comme Sandhed til Gudfrygtighed, Pontoppidan publia des ouvrages d'économie, de linguistique et des ouvrages contre les superstitions de son temps. C'était un écrivain extrêmement prolifique, qui maîtrisait plusieurs langues (le latin et l'allemand dans ses premières œuvres, plus tard le danois), mais aussi différents types de textes : il écrivit des traités scientifiques, des ouvrages d'édification et de dévotion, et publia un récit de voyage sous une forme proche du roman. 


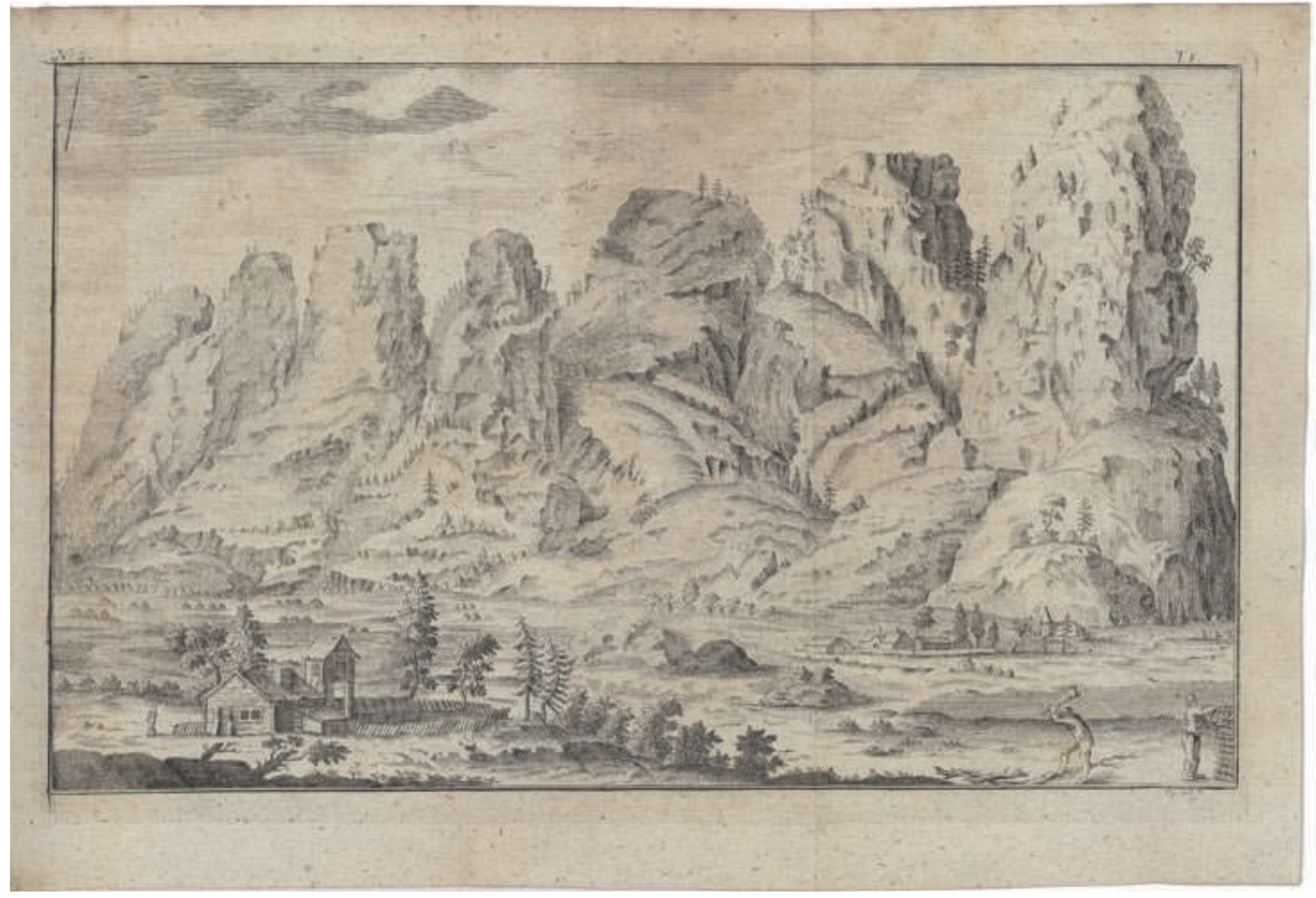

Gravure extraite du premier volume de l'Histoire naturelle de la Norvège (édition de Copenhague, 1753), représentant une chaîne de montagnes et des activités forestières (coll. BNU). 


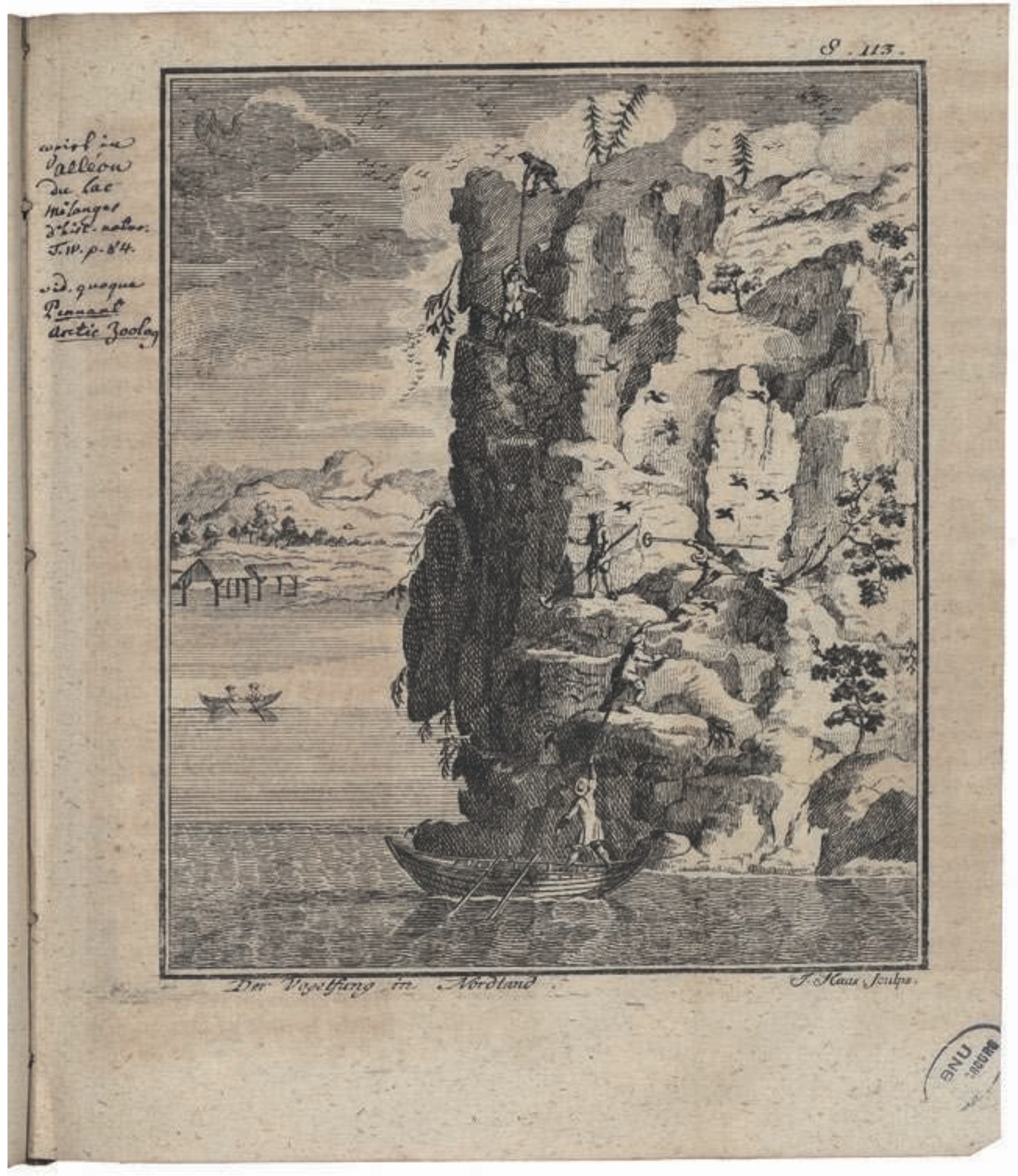

Gravure extraite du second volume de l'Histoire naturelle de la Norvège (édition de Copenhague, 1754), représentant une scène de capture d'oiseaux (coll. BNU). 
Le texte qui nous intéresse ici, le Versuch einernatürlichen Historie von Norwegen, entré à la BNU en 1873, se fonde sur l'original danois de 1752/53, Det første Forsøg paa Norges naturlige Historie ${ }^{9}$, qui fut traduit en allemand un an après sa publication (1753/54) puis, peu après, en anglais (1755). Sur plus de 800 pages, cette histoire naturelle décrit les conditions climatiques et géographiques de la Norvège ainsi que sa flore, sa faune, ses métaux, ses minéraux et sa population. L'ouvrage est richement illustré de gravures sur cuivre et comprend d'autres éléments paratextuels, comme les notes marginales et les notes de bas de page. Il contient en outre des informations sur les relations économiques de l'époque dans la double monarchie, appelle au progrès de l'agriculture et fait l'éloge du gouvernement danois. D'un bout à l'autre, le texte se fonde sur un principe physico-théologique qui conçoit les beautés de la nature, les phénomènes et les objets naturels comme des preuves de l'existence de Dieu.

S'il est impossible d'établir un lien explicite entre cette histoire naturelle et Strasbourg, l'ouvrage contient plusieurs références à la France, " si richement dotée par la Nature $"{ }^{10}$, comme le note Pontoppidan dans un passage. L'auteur utilise certains phénomènes climatiques de la France à l'appui de sa démonstration, cite dans la discussion des connaissances relatives aux minerais du pays, à sa faune, à sa flore, et se réfère fréquemment à des scientifiques français. Cela est manifeste dans l'extrait déjà cité, où il note que si Mairan avait disposé d'observations plus précises sur les aurores boréales de Norvège, son ouvrage sur ces phénomènes aurait été plus complet ; et que de même Réaumur aurait pu, dans ses Mémoires pour servir à l'histoire des insectes, décrire un nombre bien plus important d'espèces, si quelqu'un avait pu lui fournir des observations en provenance de Norvège, tant il y a là-bas d'espèces rares, que l'on ne trouve ni en Italie, ni en France, ni en Hollande. Ces termes ne sont d'ailleurs pas de Pontoppidan lui-même, mais proviennent d'une lettre que Jens Spidberg, prévôt de Christiansand, lui écrivit en 1750 et qu'il inséra ensuite dans son Histoire naturelle. La citation mentionne des chercheurs français et donne un aperçu des textes scientifiques en provenance de ce pays qui circulaient au milieu du $18^{\mathrm{e}}$ siècle en Norvège. Elle attire l'attention sur des domaines scientifiques sur lesquels on travaillait alors en France, mais qui étaient encore à l'état de friche en Norvège. Elle montre, en outre, dans quelle mesure les chercheurs français auraient pu bénéficier des apports de la Norvège pour l'accroissement de leurs connaissances. Enfin, elle souligne indirectement qu'aucun savoir exhaustif et vrai ne saurait être atteint dans la recherche sans tenir compte de la partie septentrionale de la double monarchie - aussi bien pour les phénomènes nordiques typiques que pour les questions plus générales et qu'un progrès dans la connaissance de la nature passe nécessairement par des échanges scientifiques dépassant les frontières géographiques.

Dans la suite de cet article, il ne sera toutefois pas question des connaissances sur le Nord contenues dans les traités français, ni de savoir comment les scientifiques français ont pu tirer profit de la recherche norvégienne sur la nature. Il s'agira plutôt de mettre en lumière la manière dont le savoir sur la France et les travaux des chercheurs français ont contribué à la construction d'une histoire naturelle de la Norvège. Quelle est la nature de ce savoir ? Quelle est sa fonction dans le texte ? Dans quelle mesure peut-on parler d'un " entrelacement des savoirs " et quel sens faut-il donner à cet entrelacs ?

Avec son projet de " Première histoire naturelle de la Norvège ", ainsi dénommée dans l'original danois, Pontoppidan poursuivait plusieurs objectifs : outre un éloge du Créateur, doublé d'une défense de ses propres intérêts (où les velléités autarciques de la double monarchie absolutiste et le " caméralisme " tenaient une place importante), il s'agissait pour lui d'acquérir des connaissances sur les phénomènes et objets naturels locaux. L'histoire naturelle fut ainsi écrite aussi bien pour le gouvernement que pour les cercles scientifiques dano-norvégiens et, par le biais des traductions, pour un public scientifique international, dont les représentants n'avaient le plus souvent pas accès à une connaissance directe de l'environnement norvégien.

Afin de rendre visible et tangible dans une histoire naturelle cette zone géographique de la Norvège, qui pour une grande part restait encore à décrire, il lui fallait produire un savoir. Cela fut fait de façon très concrète, sur place : d'une part en extérieur, sur le terrain, lors de ses visites annuelles d'inspection au monastère de Bergen, et d'autre part derrière un bureau, à Bergen, lors de ses " voyages " dans les volumes de sa très riche bibliothèque personnelle. Pour ce travail, l'accès à d'autres sources de savoir fut extrêmement important pour Pontoppidan : les institutions (au premier rang desquelles l'Université de Copenhague), les sociétés savantes et les bibliothèques. Mais le réseau social personnel du savant fut également crucial : il recevait continuellement des lettres d'amis et de collègues avec les dernières découvertes, mais aussi des échantillons, comme des haricots secs et des fleurs. Ces éléments de savoir recueillis dans des lettres, glanés oralement lors de ses voyages ou puisés dans les livres, ses expérimentations et son expérience directe, Pontoppidan 


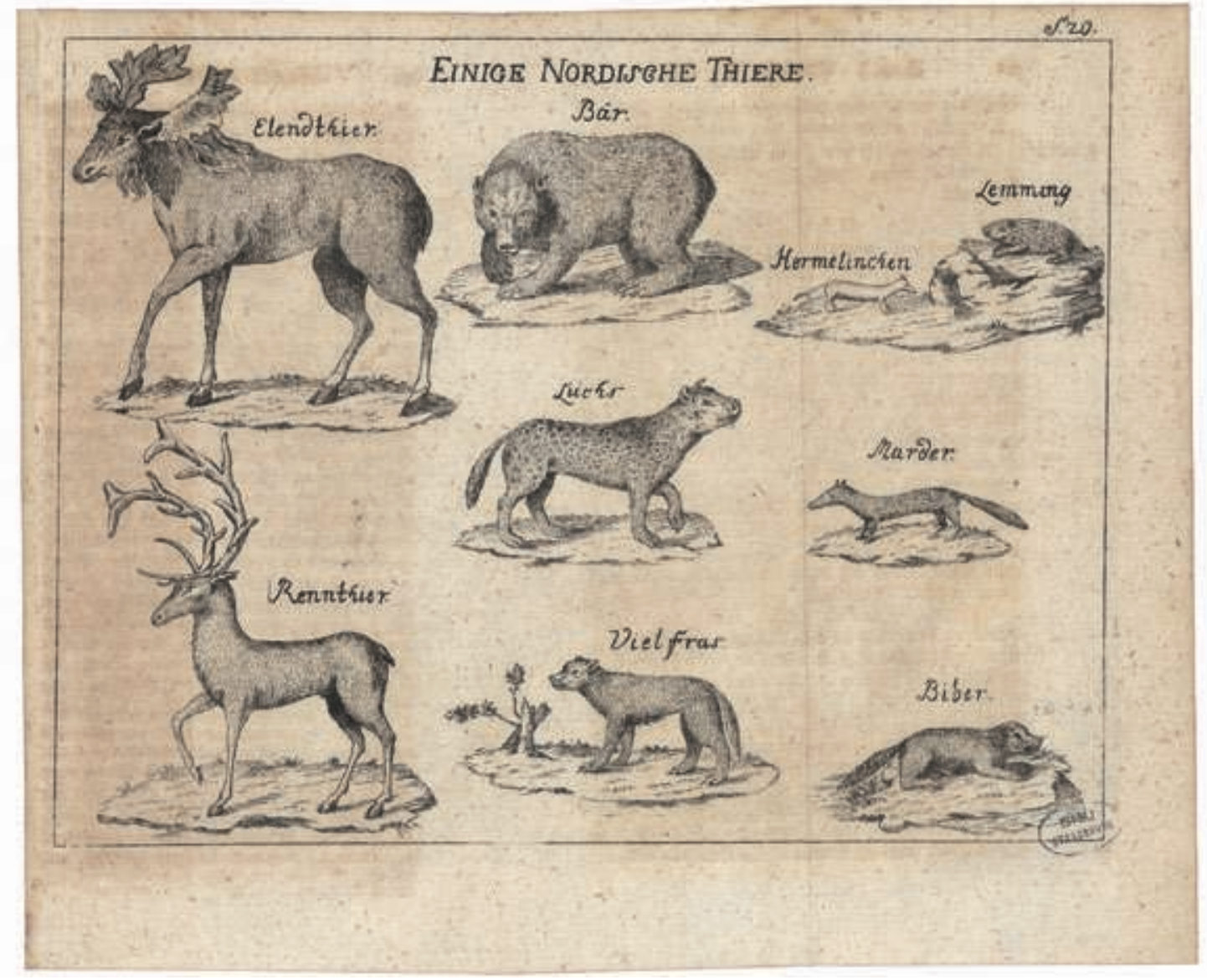

Gravure extraite du second volume de l'Histoire naturelle de la Norvège (édition de Copenhague, 1754), représentant quelques animaux des pays du Nord (coll. BNU). 


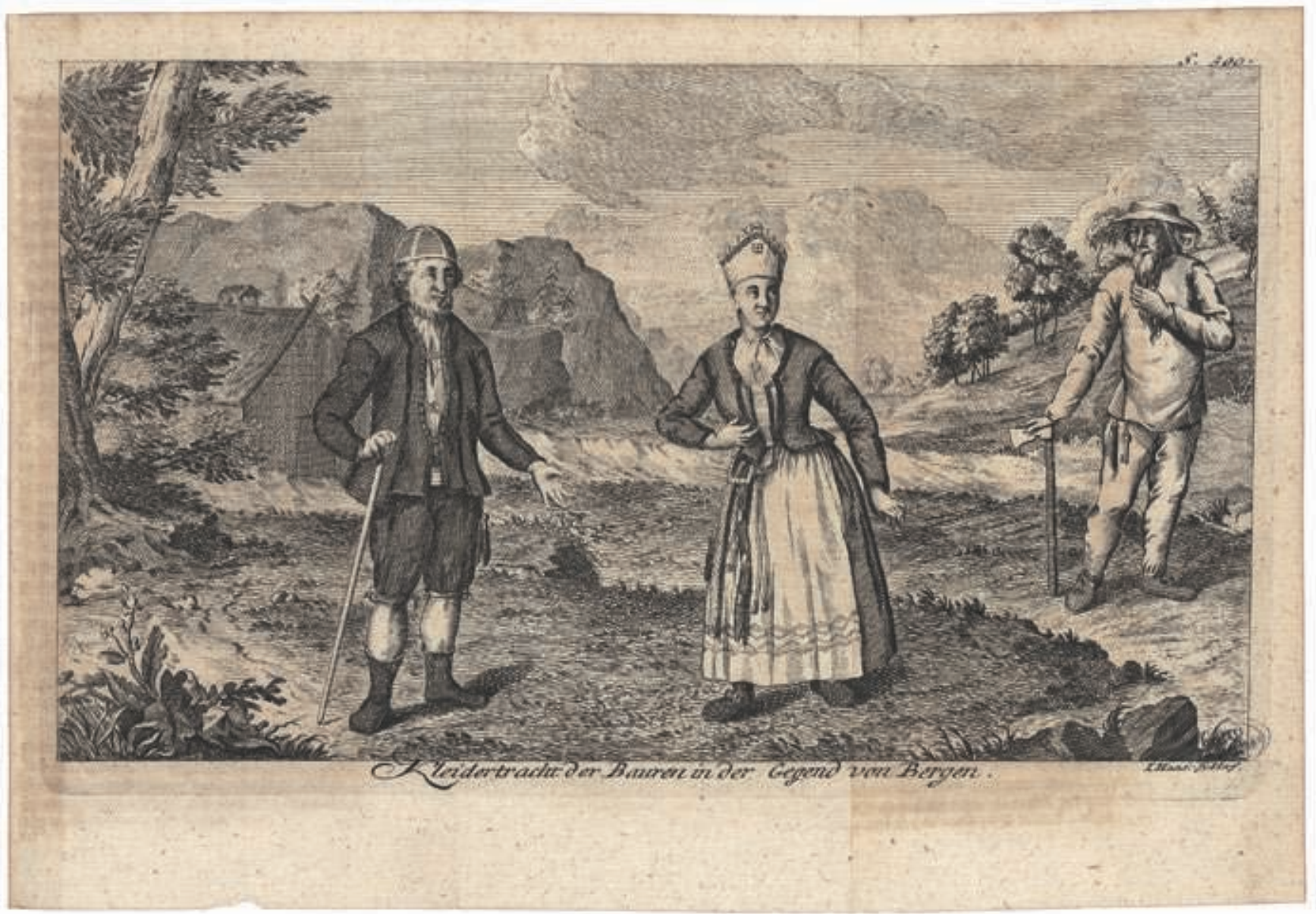

Gravure extraite du second volume de l'Histoire naturelle de la Norvège (édition de Copenhague, 1754), représentant des costumes traditionnels paysans de la région de Bergen (coll. BNU). 
les combina à travers différentes pratiques. En prenant place, au sein du " médium livre ", en tant qu'histoire naturelle de la Norvège, ce " nouveau " savoir se trouva enregistré sous forme de textes, de tableaux et d'images ; un processus qui, d'une part, a stabilisé ce savoir produit sur le terrain, et qui, d'autre part, a permis sa mobilité et sa circulation. Car en même temps, en s'inscrivant dans le circuit médiatique du livre, ce " nouveau " savoir sur la Norvège - une zone géographique encore largement inconnue - devenait pour la première fois visible et porteur de sens pour de nombreux lecteurs.

Bien que l'histoire naturelle de Pontoppidan se limite à la zone géographique norvégienne, on y trouve plusieurs références à la France, en lien avec la question de la production du savoir : parmi les quelque 150 entrées de l'index des noms de personnes de son histoire naturelle, on trouve ainsi plusieurs scientifiques français, dont certains sont ses contemporains. Au cours du processus de production d'un savoir sur les phénomènes et objets norvégiens, il a fréquemment recours à leurs textes - que ce soit pour la description générale des phénomènes et objets naturels avec les Mémoires pour servir à l'histoire des insectes (1734-1742) de René-Antoine Ferchault de Réaumur, pour les récits de voyages sur la Norvège avec l'ouvrage de Pierre Martin de la Martinière, Voyage des pais septentrionaux (1671), ou pour des introductions générales sur la Perse ou la Chine, avec le traité de Jean-Baptiste du Halde, Description géographique, historique, chronologique, politique, et physique de l'empire de la Chine et de la Tartarie chinoise (1736). Il est également fait mention de scientifiques français lors de l'analyse d'objets et de phénomènes qui ne sont pas spécifiquement norvégiens, comme dans la discussion sur le processus de fusion pendant le déluge que l'on retrouve dans l'Histoire naturelle, générale et particulière (1749-1789) de Georges-Louis Leclerc de Buffon. Pontoppidan, dans le processus de production du savoir qu'il met en œuvre pour l'histoire naturelle, utilise aussi parfois des connaissances sur la France qui émanent d'auteurs qui ne sont pas eux-mêmes français, comme Carl von Linné, qui dans le premier volume de Swenska Wetenskaps Academiens Handlingar (1739) traite des différentes périodes de développement des plantes en France et en Norvège. Il puise aussi dans des articles de revues scientifiques françaises comme la Bibliothèque raisonnée et les Mémoires de l'Académie des sciences de l'Institut de France. Enfin, une petite partie de ces connaissances venues de France, et utilisées pour générer un savoir sur le nord de la Norvège, provient de Pontoppidan lui-même (même s'il convient de préciser qu'il n'a jamais mis les pieds dans le pays) ${ }^{11}$. Ce savoir sur la France et sur les cher- cheurs français - que l'on trouve dans le texte sous forme de longues citations, de traductions et de paraphrases, principalement dans les notes de bas de page, mais aussi parfois dans le corps du texte, et qui se mêle aux autres connaissances ayant trait à l'histoire naturelle - outre qu'il vise à étendre les connaissances du lecteur, remplit deux fonctions principales. Il sert d'abord à fournir une description directe des phénomènes et objets spécifiquement norvégiens, comme dans le chapitre sur les métaux et minéraux dans lequel Pontoppidan cite plusieurs pages du carnet de voyage dans lequel le Français Martinière rapporte sa visite dans les mines des environs de Trondheim. En dépit de certains doutes (" Je ne saurais dire laquelle parmi les mines de cuivre mentionnées jusqu'ici est celle qu'observa Martinière, ce Français habitué des voyages et très curieux, et encore moins comment il peut dire avec vérité qu'à deux milles de cette mine de cuivre, l'on trouve une mine d'argent ; cela rend sa description douteuse ${ }^{12}$ ), Martinière lui permet d'expliquer de façon très exhaustive le fonctionnement d'une mine norvégienne.

Mais surtout, le savoir sur la France et les scientifiques français a pour fonction principale de confirmer la description de phénomènes ou d'objets norvégiens : il fait office de description indirecte. Comme le montrent les trois exemples suivants, cette fonction se manifeste le plus souvent dans des parallélismes au niveau du contenu, qui ne se doublent que rarement de parallèles syntaxiques, dans des analogies et des comparaisons. Ainsi lorsque, traitant de l'apparence des Norvégiens, Pontoppidan présente les agriculteurs de l'intérieur du pays comme étant de grande taille, avec des traits anguleux et, à l'inverse, les habitants des régions côtières comme corpulents, plus flegmatiques, avec des visages ronds, il s'appuie sur cette citation du Français Buffon :

"Qu'on examine dans le même canton les hommes, qui habitent les terres élevées [...], et qu'on les compare avec ceux qui occupent le milieu des vallées voisines, on trouvera que les premiers sont agiles, dispos, bienfaits, spirituels, et que les femmes y sont communément jolies, au lieu que dans le plat pays, [...] les paysans sont grossiers, pésans, malfaits, stupides, et les paysannes toutes laides ${ }^{13}$.

L'affirmation de Buffon, combinée avec la remarque de Pontoppidan, crée ici un parallélisme de contenu dont la fonction est de confirmer une observation spécifique par une observation plus générale, confirmation qui se trouve en outre encore renforcée par la répétition d'une structure syntaxique analogue. 
De la même façon, l'assertion de Pontoppidan selon laquelle les chaînes de montagnes norvégiennes " ne s'étendent pas de manière transversale, d'ouest en est, mais [...] tout le long depuis le sud jusqu'au pôle ", est d'abord suivie dans une note par un passage de l'Histoire naturelle de Buffon qui traite de l'alignement opposé de tous les autres massifs montagneux européens : "C'est en opposition aux autres types de montagnes européennes, qui en Espagne, en France, en Suisse, en Hongrie, etc. vont dans la direction de l'est ou de l'ouest ". Une autre remarque de Buffon lui fait suite, qui souligne une situation analogue sur le continent américain : " Mais en Amérique, les grandes montagnes de la Cordillère ont la même orientation que nos montagnes nordiques ". Souvent aussi, dans la discussion, des affirmations contradictoires se trouvent convoquées, principalement pour être réfutées. Par ce biais également, comme ici au moyen de l'analogie, l'exactitude d'une assertion se trouve encore plus nettement soulignée.

On trouve un autre bon exemple de l'utilisation d'une comparaison dans le but de confirmer une affirmation dans la description que fait Pontoppidan des techniques d'irrigation des agriculteurs dans le Gudbrandsdal. Ces derniers ont réalisé, au moyen de troncs d'arbres creux, " selon le rapport de Tavernier en Perse, des conduites d'eau depuis des points élevés jusqu'à ceux plus bas ". La comparaison avec la situation en Perse décrite par JeanBaptiste Tavernier dans Les six voyages apporte de la crédibilité à la description de ce système d'irrigation norvégien.

À l'aune des remarques précédentes, il est manifeste qu'il devient possible de parler, au sujet des relations entre le Versuch einer natürlichen Historie von Norwegen et la France, de véritables entrelacements des savoirs : des savoirs produits par des Français, tant sur la France que sur la Norvège ou sur d'autres régions, mais aussi des savoirs sur la France produits par Pontoppidan lui-même et par d'autres savants non français, et pour partie publiés dans des revues françaises, s'entrelacent avec des savoirs d'autres origines pour produire quelque chose de nouveau. Ils contribuent à la construction d'une histoire naturelle de la Norvège : une construction, au sens où cette histoire naturelle est tout sauf une représentation objective, mais est au contraire tout entière traversée par des intérêts personnels, religieux et politiques, surdéterminée par les réseaux sociaux de Pontoppidan et dépendante tant des conditions de production scientifique et médiatique que des moyens d'accès aux différents domaines de la connaissance dans le Bergen du milieu du $18^{\mathrm{e}}$ siècle.

À travers ces stratégies de production du savoir qui se fondent sur un entrelacement de l'inconnu et du connu que ce soit de manière comparative, par le parallélisme ou les oppositions - une zone géographique de la périphérie de l'Europe, jusque-là très imparfaitement décrite, se trouve dotée d'une histoire naturelle, elle-même sauvegardée dans le média que constitue le livre. Ces entrelacements du savoir se déploient ainsi tant dans l'espace géographique que dans l'espace médiatique du livre, et ces espaces sont en retour façonnés par ces entrelacements du savoir. Sauvegardé dans le " médium du livre ", le savoir spécifique produit localement devient capable de circuler. Il en résulte que les zones géographiques du nord de la double monarchie sont rendues visibles et compréhensibles, qu'elles deviennent porteuses de sens tant pour les lecteurs danois que - par le biais des traductions - pour les lecteurs germanophones et anglophones intéressés par la science. Grâce à ce processus d'inscription, elles se trouvent ainsi transférées de la périphérie vers le centre de la recherche - comme, entre autres, en 1873, dans les collections de la nouvelle Kaiserliche Universitäts- und Landesbibliothek de Strasbourg.

\section{Simone Ochsner}

(traduction Pierre-Brice Stahl et Julien Collonges)

\section{Notes}

1 - Pontoppidan, Erik, Versuch einer natürlichen Historie von Norwegen worinnen die Luft, Grund und Boden, Gewässer, Gewächse, Metalle, Mineralien, Steinarten, Thiere, Vögel, Fische und endlich das Naturel wie auch die Gewohnheiten und Lebensarten der Einwohner dieses Königreichs beschrieben werden aus dem Dänischen übers. von Johann Adolph Scheiben. Vol. I. Copenhague, 1753, p. 46

2 - Voir Littler, Gérard, La bibliothèque nationale et universitaire de Strasbourg, in Bulletin des bibliothèques de France, vol. 4 (2002), p. 36-46, ici p. 37. Voir aussi Dubled, Henri, Histoire de la Bibliothèque nationale et universitaire de Strasbourg, Strasbourg, 1973.

3 - Sansen, Jean, Les transformations de la Bibliothèque nationale et universitaire de Strasbourg, in Bulletin des bibliothèques de France, vol. 1 (1977), p. 25-33, ici p. 25

4 - http://www.bnu.fr/de/die-einrichtung/geschichte-der-bnu, 26.2.2013

5 - Littler, op. cit., p. 36

6 - Erik Pontoppidan et son histoire naturelle sont analysés plus en détail dans ma thèse : Ochsner Goldschmidt, Simone, Wissensspuren. Generierung, Ordnung und Inszenierung von Wissen in Erik Pontoppidans Norges naturlige Historie 1752/53, Tübingen, 2012.

7 - Nilsen, Laurits (éd.), Erik Pontoppidans Levnetsløb. Samt Brudstykker av hans Hyrdebreve, Mandal, 1897, p. 25

8 - Dahl, Gina, Bibelsk tid. Pontoppidans jordhistorie, in Bjørguin, 1 (2004), p. $67-83$, ici p. 67

9 - Pontoppidan, Erik, Det første Forsøg paa Norges naturlige Historie, forestillende dette Kongeriges Luft, Grund, Fjelde, Vande etc. og omsider Indbyggernes Naturel, samt Scedvaner og Levemaade. Vol. I et II, Copenhague, 1752/53

10 - Pontoppidan, vol. II, 1754 , p. 88

11 - Nilsen, op. cit., p. 56 sqq

12 - Pontoppidan, 1753, vol. I, p. 347

13 - Pontoppidan, 1754, vol. II, p. 445 sqq

14 - Pontoppidan, 1753, vol. I, p. 74

15 - Pontoppidan, vol. I, 1753, p. 182 